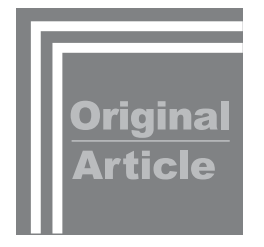

\title{
The Treatment Outcome of Transarterial Embolization for Isolated Sinus Type Dural Arteriovenous Fistula between NBCA and Onyx
}

Tasuku Imai, ${ }^{1}$ Takashi Izumi, ${ }^{1}$ Noriaki Matsubara, ${ }^{1}$ Masahiro Nishihori, ${ }^{1}$ Masashi Ito, ${ }^{1}$ Masaki Sato, ${ }^{1}$ Yosuke Tamari, ${ }^{1}$ Mamoru Ishida, ${ }^{1}$ Tetsuya Tsukada, ${ }^{1}$ Shigeru Miyachi, ${ }^{2}$ and Toshihiko Wakabayashi ${ }^{1}$

Objective: The purpose of this study was to compare the clinical outcome of transarterial embolization for isolated sinus type dural arteriovenous fistula (iDAVF) using n-butyl-cyanoacrylate (NBCA) and Onyx.

Methods: Seventeen lesions of iDAVF in 17 patients who underwent transarterial embolization between March 2008 and February 2015 were retrospectively analyzed. They were treated by embolization using NBCA in 10 patients, Onyx in 5 patients, and combination of NBCA and Onyx in 2 patients. We compared the results between embolization with NBCA and Onyx as follows: complete occlusion rate, number of embolized vessels, procedure-related complications, treatment time, dose of radiation exposure, amount of contrast agent, and total cost of the devices.

Results: The complete occlusion rate of iDAVF was significantly higher in the Onyx group: 4 of $10(40 \%)$ in NBCA and 5 of $5(100 \%)$ in Onyx $(P=0.04)$. The number of treated vessels $(5.1 \pm 1.2$ in NBCA, 1 in Onyx $[P<0.001])$, the maximum skin entrance dose (NBCA: $5030 \pm 1646$ [mGy], Onyx: $1670 \pm 564$ [mGy] in Onyx [P<0.01]), and total cost of the devices $(601,265 \pm 15,749$ [yen] in NBCA, 374,210 $\pm 122,142$ [yen] in Onyx [P <0.01]) were significantly higher in the NBCA. No significant difference was observed in the treatment time and contrast agent dose.

Conclusion: In transarterial embolization of iDAVF, a higher complete occlusion rate was obtained with Onyx, which was also advantageous for reducing the exposure dose and cost of the devices.

Keywords \ isolated sinus type dural arteriovenous fistula, n-butyl-cyanoacrylate, Onyx, transarterial embolization

\section{Introduction}

Dural arteriovenous fistula (DAVF) accompanied by cortical venous reflux presents severe neurological symptoms and unfavorable outcome, therefore, requires aggressive treatment. ${ }^{1,2}$ Although the usefulness of transvenous embolization (TVE) for the treatment of DAVF is established, TVE is

$\overline{{ }^{1} \text { Department of Neurosurgery, Nagoya University, Nagoya, Aichi, }}$ Japan

${ }^{2}$ Department of Neurosurgery, Osaka Medical College, Takatsuki, Osaka, Japan

Received: August 8, 2016; Accepted: January 5, 2017 Corresponding author: Takashi Izumi. Department of Neurosurgery, Nagoya University, 65 Tsurumai, Showa, Nagoya, Aichi 4668560, Japan

Email: my-yuzu@med.nagoya-u.ac.jp

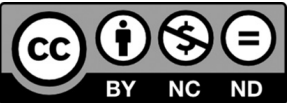

This work is licensed under a Creative Commons Attribution-NonCommercialNoDerivatives International License.

(C)2017 The Japanese Society for Neuroendovascular Therapy difficult in some cases such as isolated sinus type DAVF (iDAVF), DAVF with normal venous drainage via the affected venous sinus, and DAVF with a shunt draining directly into cortical vein. In those cases, transarterial embolization (TAE) using n-butyl-cyanoacrylate (NBCA) or particulate embolic agents has been performed. However, the radical cure rate by TAE is lower than that by TVE. Onyx Liquid Embolic System (ev3 Neurovascular, Covidien, Plymouth, MN, USA) is a liquid embolic agent with cohesive non-adhesive polymer approved for preoperative embolization in treating cerebral arteriovenous malformations in Japan. As a clinical trial of DAVF using Onyx liquid embolic system was also initiated in Japan in February 2014, its treatment would be covered the insurance in the future. We comparatively evaluated the results of TAE using NBCA and Onyx for DAVF at our hospital.

\section{Subjects and Methods}

This retrospective study was approval by our hospital's ethics committee concerning medical researches. From 
March 2008 to February 2015, 117 patients with 131 lesions of DAVF were treated endovascularly in our department. In all, 17 lesions of iDAVF in 17 patients were treated by TAE because of difficulty to access to the lesion for TVE. NBCA was used, in principle, from March 2008 to March 2014. From August 2013 to March 2014, NBCA was used as the first choice and Onyx was used concomitantly as the second choice in patients difficult to treat with NBCA alone. Onyx was used as the first choice from April 2014 to February 2015. The procedure was evaluated to be successful when the shunt flow was reduced or obliterated on cerebral angiography immediately after, compared with before the procedure. The evaluation was made by two experts of neurointervention including the operator. Background concerning iDAVF (age, sex, numbers of feeders and drainers counted in common carotid/internal carotid/ external carotid/vertebral angiograms, symptoms, bleeding on $\mathrm{CT}$, and venous congestion on MRI) and the results of treatment (complete occlusion rate, number of embolized vessels, complications, treatment time, skin entrance dose [dose of radiation exposure], amount of contrast agent, and cost of devices for the treatment) were compared between the two groups (NBCA and Onyx). Venous congestion was defined as a condition in which venous dilation was observed on MRI T2-weighted imaging and hyperintensities were observed in the areas of the brain parenchyma drained by the dilated veins on MRI FLAIR imaging. The cost of the devices was calculated by summing up the costs of all devices used for endovascular treatment including the contrast agents mentioned in the medical receipts. The procedure was performed by the transfemoral approach under local anesthesia using a biplane cerebral angiographic system (Siemens Aktiengesellschaft, Munich, Germany). The dose of radiation exposure was expressed as the estimated dose (maximum skin entrance dose) indicated by the angiographic system. The degree of occlusion was evaluated by the final cerebral angiography at the treatment, and the recurrence was evaluated by head MRI/ MRA 1 week, 3 months, and 6 months after the treatment. Patients suspected to have recurrence on MRI/MRA were examined by cerebral angiography, and patients who showed cortical venous reflux were re-treated.

\section{Statistical analysis}

All statistical analyses were performed using the statistical software EZR (Saitama Medical Center, Jichi Medical University, Saitama, Japan). Continuous variables showing normal distribution were examined by the unpaired t-test, and those showing non-normal distribution were examined by the Mann-Whitney U-test. Nominal variables were examined by Fisher's exact test. For all statistical analyses, $\mathrm{P}<0.05$ was considered statistically significant.

\section{Results}

Seventeen lesions of iDAVF in 17 patients were assigned (nine males and eight females, mean age: $68.4 \pm 6.5$ years). Patients' demographic and angiographic findings and clinical outcomes are shown in Table 1. The procedure was performed using NBCA in 10 patients, using Onyx in five patients, and using both Onyx and NBCA in two patients. The mean follow-up period was $22.7 \pm 28.3$ months (median: 4.5 months, range: $0-75$ in NBCA, 11 months, range: 6-18 in Onyx, 28 months, range: 18-38 in NBCA + Onyx), during which recurrence was observed in two patients in the NBCA. In all, 12 patients (seven in NBCA, four in Onyx, and one in NBCA + Onyx) had transverse-sigmoid sinus DAVF, and five patients (three in NBCA, one in Onyx, and one in NBCA + Onyx) had superior sagittal sinus DAVF. All patients showed cortical venous reflux, and all but one patient had symptomatic onset. Hemorrhage was noted in six patients $(40 \%)$ on CT, and venous congestion was detected in five patients (33\%) on MRI. Table 2 showed the background of the NBCA $(n=10)$ and Onyx $(n=5)$. There were no significant differences in the age, sex, number of feeders and drainers, clinical symptoms, and hemorrhage or venous congestion on radiological imaging between two groups. Table 3 designated the clinical outcomes. The procedure was successful in all patients. The complete occlusion rate on the final cerebral angiography at the treatment was significantly higher in Onyx (4/10 [40\%] in NBCA vs. $5 / 5$ [100\%] in Onyx; $\mathrm{P}=0.04)$. The number of embolized vessels differed significantly $(5.1 \pm 1.2$ in NBCA vs. 1 in Onyx; $\mathrm{P}<0.001)$ as the injection of embolization material was achieved via a single vessel in all patients in the Onyx. Procedure-related complications were encountered 4 in 17 sessions. In the NBCA group, asymptomatic vascular perforation occurred in one patient, and transient cranial nerve palsy occurred in one patient. Gluing of the microcatheter was occurred in two patients in the Onyx group. No ischemic complications related to the embolized area were observed in either group. There was no significant difference in the total treatment time (309 \pm 65.7 [min] in NBCA, $271 \pm 58.4$ [min] in Onyx; $\mathrm{P}=0.293)$ or the amount of the contrast agent $(238 \pm 52.1$ [mL] in NBCA group, $281 \pm 52.6[\mathrm{~mL}]$ in Onyx: $\mathrm{P}=0.16)$. 


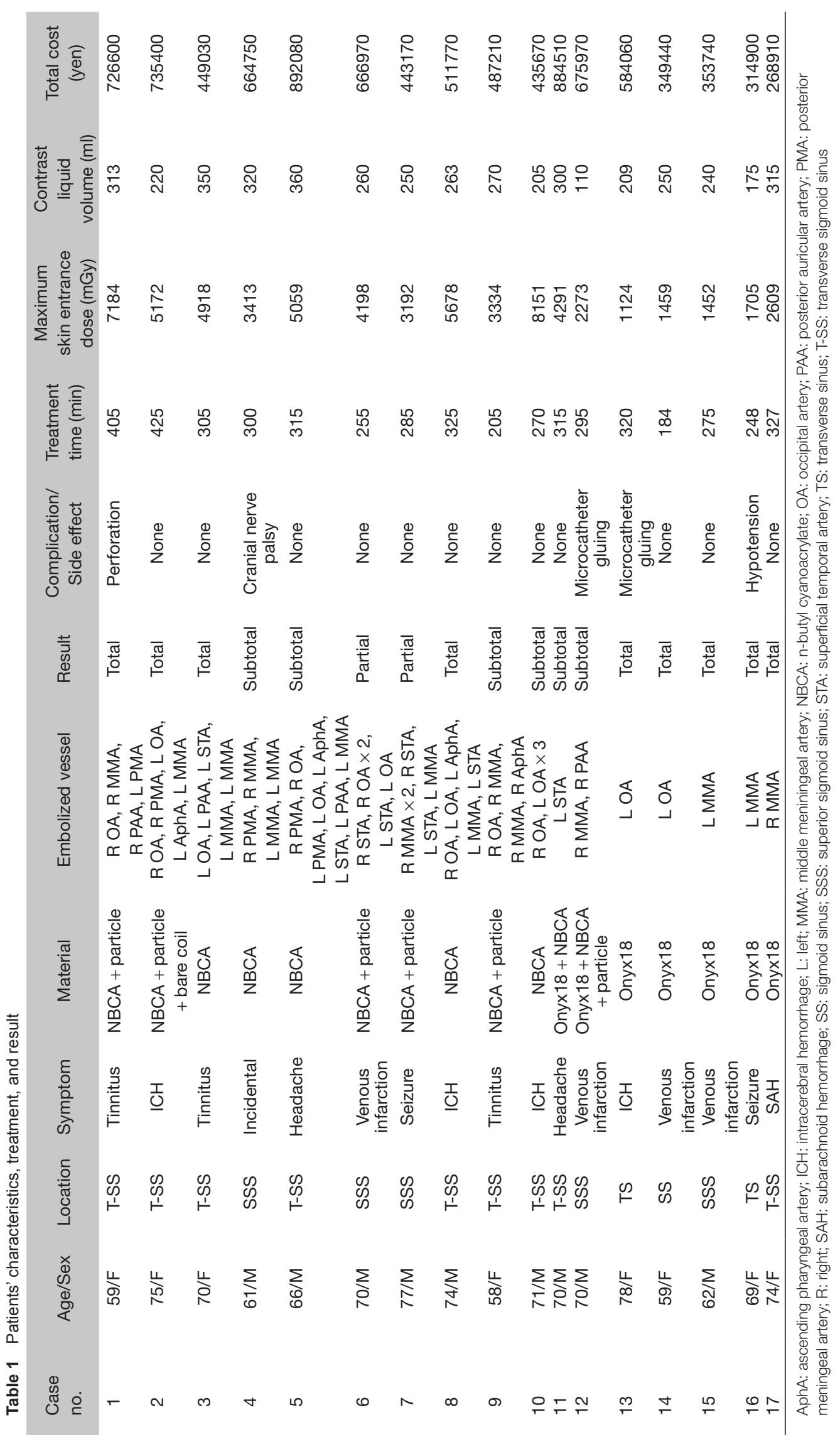


Table 2 Baseline characteristics of iDAVF treated with NBCA and Onyx

\begin{tabular}{lccc} 
& NBCA $(\mathrm{N}=10)$ & Onyx $(\mathrm{N}=5)$ & P value \\
Age (years) & $68.1 \pm 6.81$ & $68.4 \pm 7.96$ & $0.94(\mathrm{~b})$ \\
Male & $6(60 \%)$ & $1(20 \%)$ & $0.282(\mathrm{a})$ \\
Number of feeders & $7.7 \pm 2.50$ & $6.6 \pm 3.36$ & $0.485(\mathrm{~b})$ \\
Number of drainers & $3.2 \pm 0.87$ & $3.2 \pm 1.30$ & $1(\mathrm{~b})$ \\
Symptomatic & $9(90 \%)$ & $5(100 \%)$ & $1(\mathrm{a})$ \\
Hemorrhage in CT scan & $3(30 \%)$ & $3(60 \%)$ & $0.329(\mathrm{a})$ \\
Venous congestion in MRI & $3(30 \%)$ & $2(40 \%)$ & $1(\mathrm{a})$ \\
Observation period (months) & $27.8 \pm 33.9$ & $12.4 \pm 5.41$ & $0.34(\mathrm{~b})$ \\
\hline
\end{tabular}

(a) Fisher's exact test; (b) unpaired t-test; iDAVF: isolated sinus type dural arteriovenous fistula; NBCA: n-butyl-cyanoacrylate

Table 3 Comparison of clinical outcome between NBCA and Onyx

\begin{tabular}{lccc} 
& NBCA $(\mathrm{N}=10)$ & Onyx $(\mathrm{N}=5)$ & $\mathrm{P}$ value \\
Complete obliteration & $\mathrm{N}=4(40 \%)$ & $\mathrm{N}=5(100 \%)$ & $0.04(\mathrm{a})$ \\
Number of embolized arteries & $5.1 \pm 1.2$ & 1 & $<0.001(\mathrm{~b})$ \\
Complication & $\mathrm{N}=2(20 \%)$ & $\mathrm{N}=2(40 \%)$ & $1(\mathrm{a})$ \\
Treatment time (min) & $309 \pm 65.7$ & $271 \pm 58.4$ & $0.293(\mathrm{c})$ \\
Maximum skin entrance dose (mGy) & $5030 \pm 1646$ & $1670 \pm 564$ & $<0.001(\mathrm{c})$ \\
Contrast liquid volume (ml) & $281 \pm 52.6$ & $238 \pm 52.1$ & $0.16(\mathrm{c})$ \\
Total cost (yen) & $01265 \pm 15749$ & $374210 \pm 122142$ & $0.01(\mathrm{c})$ \\
Total sessions of embolization & $1.8 \pm 1.03$ & $1.4 \pm 0.54$ & $0.582(\mathrm{~b})$ \\
Recurrence & $2(20 \%)$ & $0(0 \%)$ & $0.524(\mathrm{a})$ \\
\hline
\end{tabular}

(a) Fisher's exact test; (b) Mann-Whitney U-test; (c) unpaired t-test; NBCA: n-butyl-cyanoacrylate

The maximum skin entrance dose $(5030 \pm 1649$ [Gy] in NBCA, $1670 \pm 564$ [Gy] in Onyx; $\mathrm{P}<0.01)$ and total cost of devices $(601,265 \pm 15,749$ [yen] in NBCA, 374,210 \pm 122,142 [yen] in Onyx; $\mathrm{P}<0.01$ ) were significantly higher in the NBCA. The number of treatment sessions was higher in NBCA, but the difference was not significant.

Two patients with transverse-sigmoid sinus DAVF cases were treated by the embolization, using both NBCA and Onyx. Their procedural outcomes were subtotal occlusion, without complications. The follow-up period was 38 and 18 months, respectively, and one patient had recurrence.

\section{Representative case using NBCA}

A 74-year-old male, who had a history of spinal cord injury and regularly used a wheelchair due to bilateral lower limb paralysis and right hemiparesis, consulted a local hospital due to right homonymous hemianopia and disorientation. As head CT demonstrated right parietal subcortical hemorrhage (Fig. 1A), and imaging examinations suggested DAVF, he was referred to our hospital. A diagnosis of isolated sinus type right transverse sinus DAVF was made by cerebral angiography, which also showed a large number of cortical venous refluxes including vein of Labbe (Fig. 1B). Transarterial embolization with NBCA was performed under local anesthesia. A 6 Fr long sheath was placed in the right femoral artery, and a $6 \mathrm{Fr}$ guiding catheter (Envoy, Cordis, Johnson \& Johnson, Fremont, CA, USA) was placed in the right external carotid artery, Marathon microcatheter (ev3 Neurovascular, Covidien) was guided to each feeder, and TAE was performed using $17 \%$ diluted NBCA. The amount of NBCA used was $0.07 \mathrm{~mL}$ via the right occipital artery, $0.32 \mathrm{~mL}$ via the right ascending pharyngeal artery, $0.07 \mathrm{~mL}$ via the right superficial temporal artery, $0.22 \mathrm{~mL}$ via the right posterior auricular artery, and $0.47 \mathrm{~mL}$ via the petrosquamous branch of the right middle meningeal artery. A guiding catheter was led to the left external carotid artery and Marathon was advanced to the left occipital artery, and subsequently, $0.57 \mathrm{~mL}$ of $17 \%$ diluted NBCA was injected resulting in obliteration of the shunt flow (Figs. 1D-1F). After the procedure, no new neurological findings appeared, but as right homonymous hemianopia and disorientation associated with subcortical hemorrhage persisted, he was discharged to a rehabilitation hospital.

\section{Representative case using Onyx}

A 69-year-old woman presented with seizure, aphasia, and right hemiparesis and was transported to a local hospital. Head MRI demonstrated venous edema and subarachnoid hemorrhage (Figs. 2A-2C). Cerebral angiography indicated isolated sinus type left transverse sinus DAVF with reflux to vein of Labbe (Fig. 2D). After the palliative 

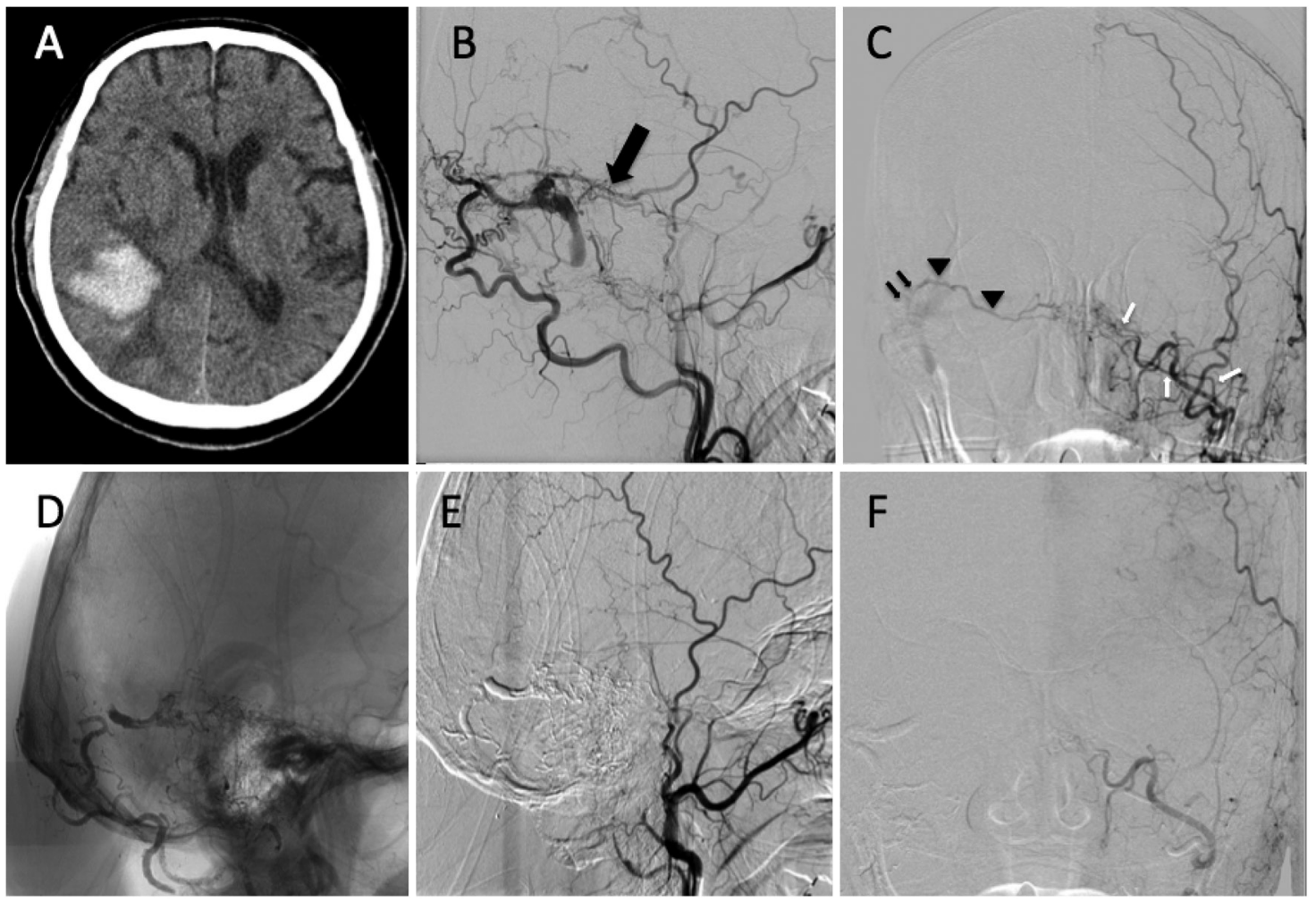

Fig. 1 A 74-year-old male presented with right hemianopsia and disorientation. (A) Conventional CT axial image shows a high intensity lesion, $43 \mathrm{~mm}$ in the maximum diameter, in the right parietal lobe. (B) Lateral view of right ECA angiogram shows an isolated sinus dural arteriovenous fistula of the right transverse sinus with reflux to the vein of Labbe (black arrow) and some cortical veins. (C) AP view of left ECA angiogram presents that left OA branch (white arrow) connects the right OA (black triangle) and feeds to the fistula (black double arrow). (D) NBCA cast in a spot image just after TAE. (E) Lateral view of right ECA angiogram just after TAE shows complete occlusion. (F) AP view of left ECA angiogram just after the procedure indicates complete occlusion of the feeder of the left OA. AP: anteroposterior; ECA: external carotid artery; NBCA: n-butyl-cyanoacrylate; OA: occipital artery; TAE: transarterial embolization

transarterial embolization of the left occipital artery and ascending pharyngeal artery using NBCA and particles, she was transferred to our hospital. Transarterial embolization with Onyx was performed under local anesthesia. A 6 Fr long sheath was placed in the right femoral artery, a $6 \mathrm{Fr}$ guiding catheter (Slimguide, Medikit, Tokyo, Japan) was placed in the left external carotid artery, and Marathon microcatheter was selectively guided to the posterior convexity branch of the left middle meningeal artery using a type 1014 TENROU guidewire (Kaneka Medics, Osaka, Japan), and $1.16 \mathrm{~mL}$ of Onyx 18 was injected, resulting in complete occlusion (Figs. 2E and 2F). Persistent hypotension, considered to be an adverse reaction to dimethyl sulfoxide (DMSO), was observed after the treatment for 14 days. Aphasia and right hemiparesis, which were observed preoperatively, were gradually improved, and the patient was discharged for rehabilitation with score 2 on the modified Rankin scale. Complete occlusion was maintained on cerebral angiography performed 6 months after the treatment.

\section{Discussion}

DAVF accompanied by cortical venous reflux presented severe neurological symptoms in $15 \%$ of patients and the annual mortality rate is $10.4 \%$, the annual incidence of intracranial hemorrhage is $8.1 \%$, and the incidence of non-hemorrhagic neuropathy is $6.9 \%$, all with a poor prognosis. ${ }^{1,2)}$ Direct surgical procedures are effective for the treatment of non-sinus type DAVF (Cognard type III), such as DAVF at the anterior cranial base or in the tentorial region, ${ }^{3,4)}$ and TAE is unlikely to be the first choice because TAE is usually performed via the feeding arteries from the internal carotid artery. However, TAE is selected for the treatment of iDAVF accompanied by cortical venous reflux because direct surgery is invasive and TVE is difficult to obtain the access to affected venous sinus. ${ }^{2,5,6)}$ Although NBCA has been used as an embolic material for TAE, the complete occlusion rate was $33 \%-56 \%,{ }^{7,8)}$ which was not satisfactory. Recently, there have been a number of reports 

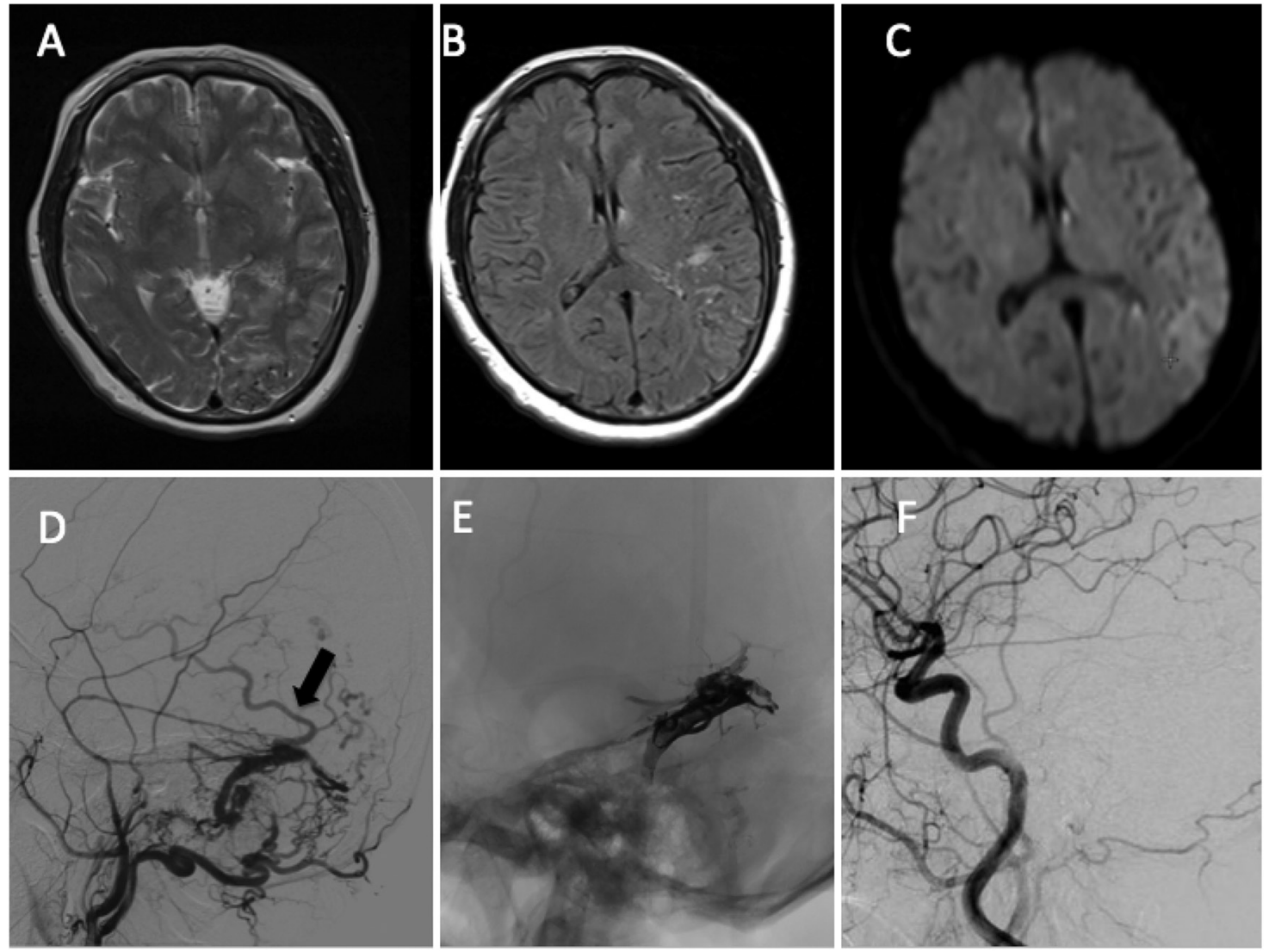

Fig. 2 A 69-year-old woman presented with seizure, aphasia, and right hemiparesis. (A) MRI T2-weighted image shows multiple flow voids in the occipital lobe. (B) MRI-FLAIR image indicated subarachnoid hemorrhage in the left parietal and temporal lobes. (C) Hyperintensity in the left parietal region in MRI diffusion-weighted image with no significant abnormal intensity in the corresponding area in FLAIR suggests venous congestion or early ischemic change in the temporoparietal region. (D) Lateral view of left ECA angiogram shows an isolated sinus dural arteriovenous fistula of the left transverse sinus with reflux to the vein of Labbe (black arrow). (E) Onyx cast spot image just after transarterial embolization. (F) Lateral view of CCA angiogram 6 months after the Onyx embolization shows complete occlusion and no recurrence. CCA: common carotid artery; ECA: external carotid artery

of using Onyx for the treatment of DAVF ${ }^{910)}$ with more favorable complete occlusion rates of $70 \%-95 \%{ }^{10-13)}$

\section{Advantage of transarterial embolization with Onyx}

Because Onyx is less adhesive liquid embolus and longer reflux from the tip of microcatheter could be possible, it can make sufficient proximal flow arrests and enable distal penetration. ${ }^{14,15)}$ Also, as it is less likely to be fragmented compared with NBCA, it can penetrate retrogradely into other feeders and drainers from the shunt point by one injection. These characteristics of Onyx are considered to contribute to the high complete occlusion rates. ${ }^{16)}$ Chandra et al. reported that complete occlusion could be achieved by a single procedure using Onyx in $85 \%$ of the patients. ${ }^{11)}$ By guiding the microcatheter to near the shunt point and wedging it into thin feeders, a high embolization rate can be achieved,9) and skin necrosis caused by embolization via subcutaneous vessels, such as the superficial temporal and occipital arteries, can be avoided.

\section{Procedure-related complication (Gluing of a microcatheter)}

Although Onyx is less adhesive and more controllable than NBCA, it must be injected with reflux from the tip of microcatheter, and the removal of the microcatheter may become difficult with prolongation of the duration of injection. The frequency of the occurrence of gluing of a microcatheter has been reported to be $2.5 \%-3.2 \% .^{14,16)}$ If it happens in cerebral arteriovenous malformation, there is a risk of brain parenchymal hemorrhage, ${ }^{17)}$ but this risk is low in dural arteriovenous fistula (DAVF), which is usually approached via the arteries from external carotid artery or dural artery.9) However, the risk of ischemic complications increases if 
the catheter could be glued in the common carotid artery. Gluing of a microcatheter was occurred in two cases in the early part of this study, and the microcatheter was retrieved using an endovascular snare device. Measures to prevent gluing of a microcatheter include the use of a microcatheter with a detachable tip, ${ }^{18)}$ shortening the injection time, concomitant use of a distal access catheter, and use of a larger diameter guiding catheter for the concomitant use of a coaxial endovascular snare device. Recently, there have also been a number of reports of injection of Onyx using a dual lumen balloon catheter (Sceptor C, Microvention, Tustin, CA, USA). It has been reported that Onyx using a balloon catheter in TAE for iDAVF reduced the total procedure time, Onyx injection time, and radiation exposure dose with improved complete occlusion rate. In addition, the risk of gluing of a microcatheter can be reduced compared with the use of a usual microcatheter. ${ }^{19,20)}$ Although TAE using Onyx still remains outside insurance coverage in Japan, it is considered a useful technique.

\section{Procedure-related complication (cranial nerve palsy)}

In TAE for DAVF using Onyx, the occurrence of cranial nerve palsy is $5.6 \%-12.3 \%$, which is higher than that using NBCA. ${ }^{16)}$ Particularly, the incidence of trigemino-cardiac reflex, which may cause bradycardia or cardiac arrest, has been reported to be $11.1 \% .{ }^{21)}$ This reflex has been reported frequently when Onyx was injected via the middle meningeal artery. ${ }^{21-23)}$ The frequent occurrence of cranial nerve palsy after TAE using Onyx may be explained by neurotoxicity of $\mathrm{DMSO}^{21,22)}$ and the facilitated permeation of Onyx to smaller vessels by the plug and push technique. ${ }^{24)}$ These complications of TAE using Onyx are considered to be avoided by placing the microcatheter as close to the shunt point as possible, paying attention to reflux, and monitoring the injection by a whole team consisting of not only the operator, but also assistants those are proficient in procedures using Onyx and versed in anatomy. Also, as there is a report that trigemino-cardiac reflex occurred in $33 \%$ of the patients who underwent TVE using Onyx and that all patients who suffered trigemino-cardiac reflex had cavernous sinus DAVF, ${ }^{21)}$ treatments of DAVF of the cavernous sinus, which is rich in cranial nerve distribution, using Onyx should be carefully determined.

\section{Radiation exposure and cost of the devices}

The results of this study that the dose of radiation exposure and cost of the devices were lower with Onyx may lead to alleviation of the patients' economic and physical burdens. In TAE using NBCA, which is performed under digital subtraction angiography, radiation exposure increases with the number of embolized vessels, and angiography for confirmation is necessary before and after injection of the embolic material. With Onyx, on the other hand, the duration of radiographic monitoring during the treatment is longer, but the embolic material can be injected under subtracted fluoroscopic monitoring (blank road mapping). This is considered to have contributed to the control of radiation exposure, leading to the significant difference in the skin entrance dose in this study while there was no difference in the total treatment time between the two groups. With NBCA, TAE of multiple other feeders must be performed before TAE of the target vessel to reduce residual cortical venous refluxes, and the consequent increase in the number of catheters used is regarded to have resulted in an increase in the total cost of the devices. In patients with iDAVF, which is difficult to treat by TVE, palliative TAE using NBCA or a particulate embolic material was often inevitably selected. Therefore, the accomplishment of complete occlusion of the shunt point and multiple feeders by a few attempts of TAE with retrograde injection of Onyx is quite unique.

\section{Consideration of transarterial embolization with Onyx}

Transarterial embolization with Onyx is considered to be applicable to cortical venous refluxes without drainage into sinuses (Cognard type III) in addition to iDAVF. Onyx is most effective when used in such pathological conditions. If, however, feeders are involved in the cranial nerves, the risk of cranial nerve palsy is higher with Onyx than with NBCA, so the appropriate selection of patients with sufficient preprocedural evaluation of the risk of cranial nerve palsy is important. In addition, TAE using Onyx is usually not recommended for patients in whom the dural arterial supply drains directly into dural venous sinuses because of the risk of outflow obstruction resulting in venous congestion or venous infarction. Thus, while Onyx is useful, it must be recognized that the selection of patients with careful evaluation is important and that the procedure requires sufficient technical proficiency and knowledge of cerebral artery and venous anatomy.

\section{Limitations}

The number of patients evaluated in this study was only 15 and was insufficient for comparison of two groups. In addition, there may have been treatment experience bias since 
TAE was performed primarily using NBCA in the first half and Onyx in the second half of the study period. Moreover, some patients were performed palliative TAE at another hospital before transfer to our hospital.

\section{Conclusion}

On comparison of TAE using NBCA or Onyx for iDAVF, a high complete occlusion rate could be achieved using Onyx with fewer embolized vessels and less radiation exposure and cost. Therefore, TAE using Onyx is considered to be advantageous for reducing both physical stress and medical cost.

\section{Disclosure Statement}

Takashi Izumi is given a manuscript fee from Covidien Japan Inc., and a research fund from Kaneka Corporation. Covidien Japan Inc. is related to an embolic material evaluated in this study. The others have no conflicts of interest.

\section{References}

1) van Dijk JM, terBrugge KG, Willinsky RA, et al: Clinical course of cranial dural arteriovenous fistulas with longterm persistent cortical venous reflux. Stroke 2002; 33: 1233-1236.

2) Borden JA, Wu JK, Shucart WA: A proposed classification for spinal and cranial dural arteriovenous fistulous malformations and implications for treatment. J Neurosurg 1995; 82: 166-179.

3) Lucas CP, Zabramski JM, Spetzler RF, et al: Treatment for intracranial dural arteriovenous malformations: a metaanalysis from the English language literature. Neurosurgery 1997; 40: 1119-1130; discussion 1130-1132.

4) Lawton MT, Sanchez-Mejia RO, Pham D, et al: Tentorial dural arteriovenous fistulae: operative strategies and microsurgical results for six types. Neurosurgery 2008; 62: 110-124; discussion 124-125.

5) Komiyama M, Ishiguro $T$, Matsusaka $Y$, et al: Transfemoral, transvenous embolisation of dural arteriovenous fistula involving the isolated transverse-sigmoid sinus from the contralateral side. Acta Neurochir (Wien) 2002; 144: 1041-1046; discussion 1046.

6) Hanaoka M, Satoh K, Satomi J, et al: Microcatheter pull-up technique in the transvenous embolization of an isolated sinus dural arteriovenous fistula. Technical note. J Neurosurg 2006; 104: 974-977.

7) Miyamoto N, Naito I, Shimizu T, et al: Efficacy and limitations of transarterial acrylic glue embolization for intracranial dural arteriovenous fistulas. Neurol Med Chir (Tokyo) 2015; 55: 163-172.

8) Rabinov JD, Yoo AJ, Ogilvy CS, et al: ONYX versus n-BCA for embolization of cranial dural arteriovenous fistulas. J Neurointerv Surg 2013; 5: 306-310.

9) Stiefel MF, Albuquerque FC, Park MS, et al: Endovascular treatment of intracranial dural arteriovenous fistulae using Onyx: a case series. Neurosurgery 2009; 65: 132-139; discussion 139-140.

10) Cognard C, Januel AC, Silva NA, et al: Endovascular treatment of intracranial dural arteriovenous fistulas with cortical venous drainage: new management using Onyx. AJNR Am J Neuroradiol 2008; 29: 235-241.

11) Chandra RV, Leslie-Mazwi TM, Mehta BP, et al: Transarterial onyx embolization of cranial dural arteriovenous fistulas: long-term follow-up. AJNR Am J Neuroradiol 2014; 35: 1793-1797.

12) Nogueira RG, Dabus G, Rabinov JD, et al: Preliminary experience with onyx embolization for the treatment of intracranial dural arteriovenous fistulas. AJNR Am J Neuroradiol 2008; 29: 91-97.

13) Lv X, Jiang C, Li Y, et al: Embolization of intracranial dural arteriovenous fistulas with Onyx-18. Eur J Radiol 2010; 73: 664-671.

14) Lv X, Jiang C, Zhang J, et al: Complications related to percutaneous transarterial embolization of intracranial dural arteriovenous fistulas in 40 patients. AJNR Am J Neuroradiol 2009; 30: 462-468.

15) Rezende MT, Piotin M, Mounayer C, et al: Dural arteriovenous fistula of the lesser sphenoid wing region treated with Onyx: technical note. Neuroradiology 2006; 48: 130-134.

16) Lv X, Jiang C, Li Y, et al: Results and complications of transarterial embolization of intracranial dural arteriovenous fistulas using Onyx-18. J Neurosurg 2008; 109: 1083-1090.

17) Gore P, Theodore N, Brasiliense L, et al: The utility of onyx for preoperative embolization of cranial and spinal tumors. Neurosurgery 2008; 62: 1204-1211; discussion 1211-1212.

18) Maimon S, Strauss I, Frolov V, et al: Brain arteriovenous malformation treatment using a combination of Onyx and a new detachable tip microcatheter, SONIC: short-term results. AJNR Am J Neuroradiol 2010; 31: 947-954.

19) Chiu AH, Aw G, Wenderoth JD: Double-lumen arterial balloon catheter technique for Onyx embolization of dural arteriovenous fistulas: initial experience. J Neurointerv Surg 2014; 6: 400-403.

20) Kim JW, Kim BM, Park KY, et al: Onyx embolization for isolated type dural arteriovenous fistula using a dual-lumen balloon catheter. Neurosurgery 2016; 78: 627-636. 
21) Lv $\mathrm{X}, \mathrm{Li} \mathrm{Y}$, Jiang $\mathrm{C}$, et al: The incidence of trigeminocardiac reflex in endovascular treatment of dural arteriovenous fistula with onyx. Interv Neuroradiol 2010; 16: $59-63$.

22) Ong CK, Ong MT, Le K, et al: The trigeminocardiac reflex in Onyx embolisation of intracranial dural arteriovenous fistula. J Clin Neurosci 2010; 17: 1267-1270.
23) Wang J, Wu HC, Wang WW, et al: Trigeminal cardiac reflex caused by onyx embolization of intracranial dural arteriovenous fistula. Turk Neurosurg 2016; 26: 325-3230.

24) Li C, Wang Y, Li Y, et al: Cranial nerve dysfunction associated with cavernous dural arteriovenous fistulas after transvenous embolization with onyx. Cardiovasc Intervent Radiol 2015; 38: 1162-1170. 